\title{
CARACTERIZAÇÃO DE MAPAS LEGADOS DE SOLOS: USO DE INDICADORES EM MAPAS COM DIFERENTES ESCALAS NO RIO GRANDE DO SUL ${ }^{(1)}$
}

\author{
Eliana Casco Sarmento ${ }^{(2)}$, Elvio Giasson ${ }^{(3)}$, Eliseu José Weber ${ }^{(4)}$, Carlos Alberto Flores ${ }^{(5)}$, \\ David Goodrich Rossiter ${ }^{(6)}$ \& Heinrich Hasenack ${ }^{(3)}$
}

\begin{abstract}
RESUMO
Mapas convencionais de solos têm adquirido importância crescente como entrada para diversas aplicações, muitas vezes sem levar em conta a sua qualidade deles. $O$ objetivo deste estudo foi descrever e comparar mapas de solos usando indicadores quantitativos de fácil determinação. Foram utilizados nove mapas elaborados em diferentes escalas no Rio Grande do Sul e o software ArcGIS. Calcularam-se a escala efetiva, o número de polígonos menores que a área mínima mapeável e um índice de complexidade de forma, e quantificaram-se o número de classes taxonômicas, de tipos de solos e de unidades de mapeamento e a fração da área com dados não uniformes. Os resultados evidenciaram que a qualidade de mapas de solos tende a ser inferior ao presumido para sua escala de apresentação, o que demonstra a importância e a necessidade de avaliações prévias ao seu uso. Indicadores computados a partir dos polígonos e da legenda possibilitam avaliação rápida e objetiva, com potencial de aplicação na sistematização e documentação de dados legados de solos no Brasil.
\end{abstract}

Termos de indexação: mapas de solos, avaliação, escala, qualidade, adequação.

(1) Parte da Tese de Doutorado do primeiro autor. Recebido para publicação em 7 de abril de 2014 e aprovado em 19 de agosto de 2014.

(2) Doutoranda em Ciência do Solo, Universidade Federal do Rio Grande do Sul - UFRGS. Av. Bento Gonçalves, 7712. CEP 91540000 Porto Alegre (RS). Bolsista da CAPES. E-mail: eliana.sarmento@ufrgs.br

(3) Professor, UFRGS. E-mail: giasson@ufrgs.br, hhasenack@ufrgs.br

(4) Pesquisador Associado, UFRGS. E-mail: eliseu.weber@ufrgs.br

(5) Pesquisador, Embrapa Clima Temperado. BR 392, km 78. Caixa Postal 403. CEP 96001-970 Pelotas (RS). E-mail: carlos.flores@embrapa.br

(6) Adjunct Associate Professor, Department of Crop and Soil Sciences, Cornell University. 14853 Ithaca (NY), USA. E-mail: dgr2@cornell.edu 


\title{
SUMMARY: CHARACTERIZATION OF LEGACY SOIL MAPS: USE OF INDICATORS IN MAPS AT DIFFERENT SCALES IN RIO GRANDE DO SUL
}

\begin{abstract}
Conventional soil maps have acquired increasing importance as inputs for various applications, often without regard to their quality. The aim of this study was to assess and compare soil maps using easily determinable quantitative indicators. Nine soil maps (at different scales, from Rio Grande do Sul) and ArcGIS software were used. Effective scale number (NSN), polygons smaller than the minimum legible delineation $(M L D)$, shape complexity index (S), number of taxonomic classes, soil types, mapping units, and proportion of areas with non-uniform data (PND) were computed. Results showed that the quality of legacy soil maps tends to be lower than presumed for their presentation scale, what shows the importance and need for quality assessments prior to using them. Indicators computed from polygons and map legend enable quick and objective assessment, with potential application in systematizing and documenting legacy soil data in Brazil.
\end{abstract}

Index terms: soil maps, assessment, scale, quality, adequacy.

\section{INTRODUÇÃO}

Um levantamento de solos é uma estratégia científica com base nos conceitos de fatores de formação de solos associados com relações solo-paisagem (Hudson, 1992). Os levantamentos convencionais contemplam o estudo do terreno e das características de perfis representativos, compreendendo descrição morfológica, caracterização física e química, classificação taxonômica e espacialização (mapeamento) da ocorrência dos diferentes tipos de solos. Assim, os mapas de solos são a materialização de um conhecimento estruturado sobre a distribuição dos solos na paisagem (Bui, 2004).

Os levantamentos de solos são enquadrados em diferentes níveis de detalhamento, cada qual com recomendações para a densidade de observações e amostragens em campo e para a escala de apresentação do mapa final. Os dois primeiros admitem variações em razão das características da área e da experiência da equipe e podem ser substancialmente reduzidos com o uso de geotecnologias (IBGE, 2007). A escala, contudo, costuma ser determinada em função dos objetivos do levantamento e do material usado como base para delimitar as unidades de mapeamento, entre outros fatores. Todavia, sendo um processo manual, a delimitação dificilmente segue normas cartográficas estritas quanto à escala escolhida e aos aspectos a ela relacionados, como a acurácia posicional, por exemplo (Hengl \& Husnjak, 2006; Nussbaum et al., 2011).

De fato, o mapeamento enfrenta o desafio de transcrever a variação tridimensional contínua dos solos e de suas propriedades na paisagem para fronteiras bidimensionais discretas que delimitam a ocorrência de classes taxonômicas. Há também a dificuldade prática de manter uniformidade durante o processo, evitando a criação de polígonos com dimensão inferior à área mínima mapeável em algumas partes do mapa e generalização excessiva em outras. Como consequência, mapas convencionais de solos inevitavelmente contêm uma série de imperfeições e incertezas, decorrentes tanto de limitações inerentes à própria forma de representação cartográfica quanto de eventuais falhas ou lacunas nas amostragens em campo (Heuvelink \& Webster, 2001; Malone et al., 2012; Miller, 2012; Phillips, 2013).

Quanto mais detalhado for o levantamento de solos, menores tendem a ser as imperfeições e incertezas inerentes ao mapa e mais homogêneas tendem a ser as unidades de mapeamento. Em contrapartida, o esforço, o tempo e o custo para a execução das atividades de campo, as análises em laboratório e a elaboração do próprio mapa crescem consideravelmente (Nussbaum et al., 2011). Devido a esses aspectos, em contraste com as áreas a serem cobertas, a maioria dos levantamentos existentes foi executada com baixo nível de detalhamento, resultando em mapas de solos em escalas pequenas (McBratney et al., 2003; Sanchez et al., 2009). Todavia, apesar das limitações, eles ainda constituem o melhor, senão o único, registro disponível sobre a ocorrência e distribuição dos solos em muitos locais, e mais recentemente passaram a receber a denominação de "mapas legados" (Carré et al., 2007).

Nos últimos anos, dados legados de solos têm adquirido importância crescente como entrada para diversas aplicações, com destaque para estudos em mapeamento digital de solos e em modelagem ambiental (Omuto et al., 2013). Frequentemente os polígonos dos mapas convencionais de solos são convertidos do modelo vetorial para o formato de grade (raster) em SIG, sendo a resolução espacial definida em razão de outros dados raster disponíveis, como o SRTM (Shuttle Radar Topography Mission) ou imagens NDVI/MODIS (Normalized Difference Vegetation Index/Moderate Resolution Imaging Spectroradiometer). No entanto, essa resolução pode resultar excessivamente fina ou grosseira para o detalhe espacial e temático que o mapa de solos 
efetivamente possui (Nussbaum et al., 2011; Miller, 2012).

Em mapeamento digital de solos, a resolução espacial pode ser usada como recurso tanto para incrementar o detalhe quanto para generalizar mapas convencionais existentes, com métodos que possibilitem controlar o processo e obter resultados consistentes (Malone et al., 2012; Nauman \& Thompson, 2014). Em outras aplicações, entretanto, especificações inadequadas para a resolução espacial e outros parâmetros podem violar pressupostos e gerar resultados enganosos, levando a interpretações equivocadas. Assim, conhecer as características do mapa convencional torna-se fundamental para orientar o uso correto de qualquer informação dele extraída ou derivada (Carré et al., 2007; Basayigit \& Senol, 2008).

A preocupação quanto à avaliação de mapas de solos para suprir informações a diferentes usos existe há várias décadas. Beckett \& Burrough (1971) propuseram avaliar a qualidade dos mapas a partir da legenda, da exatidão da localização espacial dos limites dos polígonos e da pureza e uniformidade das unidades de mapeamento, a última modificada posteriormente por Bie \& Beckett (1973). Forbes et al. (1987) e Rossiter (2000) introduziram o conceito de adequação, considerando: escala e textura do mapa; legenda; qualidade do mapa base; e verdade de campo, ou acurácia temática. Hunter et al. (2003) discutiram a "usabilidade" de dados geoespaciais em um contexto mais amplo, considerando basicamente: qualidade dos dados (acurácia, completeza, consistência lógica); forma(to); acessibilidade/preço; e qualidade dos metadados.

Hengl \& Husnjak (2006) apresentaram a diferença entre os conceitos de qualidade, adequação e usabilidade. A primeira envolve um conjunto de características eminentemente técnicas, a segunda depende do contexto do problema a ser resolvido e a terceira considera os dois em relação à satisfação das necessidades do usuário. Com base nessas abordagens, os autores propuseram 10 indicadores, com graus variados de complexidade, computados a partir dos mapas digitalizados, dos respectivos relatórios de levantamento e de dados de referência coletados em campo.

O objetivo deste estudo foi descrever e comparar um conjunto de mapas convencionais de solos do Rio Grande do Sul usando indicadores quantitativos computados diretamente a partir dos mapas digitalizados e de suas legendas. A hipótese é que a escala de apresentação ou o nível de detalhe do levantamento não são suficientes para caracterizar a real qualidade de mapas legados de solos. Pressupõe-se que tais indicadores forneçam medidas objetivas sobre as características geométricas e temáticas dos mapas existentes, que sirvam de subsídio para a tomada de decisão consciente sobre seu uso.

\section{MATERIAL E MÉTODOS}

A área de estudo compreende a totalidade do Estado do Rio Grande do Sul e algumas áreas específicas de seu território, onde existem informações de levantamentos de solos executados no passado. As coordenadas extremas situam-se entre as latitudes $27^{\circ} 05^{\prime} \mathrm{S}$ e $33^{\circ} 45^{\prime} \mathrm{S}$ e as longitudes $49^{\circ} 41^{\prime} \mathrm{O}$ e $57^{\circ} 38^{\prime} \mathrm{O}$ (Figura 1). Predomina no Estado o clima subtropical úmido com verões quentes (Cfa, conforme Köppen) e com verões amenos (Cfb) nas porções mais elevadas. A geologia compreende uma diversidade de litologias que incluem rochas graníticas do Eon Arqueano, areníticas e basálticas da Era Mesozoica e depósitos sedimentares da Era Cenozoica. O relevo varia de plano a escarpado e apresenta altitudes que variam de menos de $10 \mathrm{~m}$ a mais de $1.000 \mathrm{~m}$. Essas litologias, altitudes e declividades integradas geram diversas formas do relevo, as quais correspondem a várias unidades geomorfológicas (IBGE, 1986). A vegetação é representada por florestas ombrófilas e estacionais e por formações campestres arbustivas e graminosas.

O material utilizado neste estudo compreende nove mapas de solos disponíveis em meio digital em formatos compatíveis com uso em SIG. Os mapas foram produzidos por meio de levantamentos convencionais em diferentes épocas e níveis de detalhamento, cobrem uma variedade de extensões geográficas e paisagens e foram elaborados em quatro escalas de apresentação (Figura 1, Quadro 1 ). O

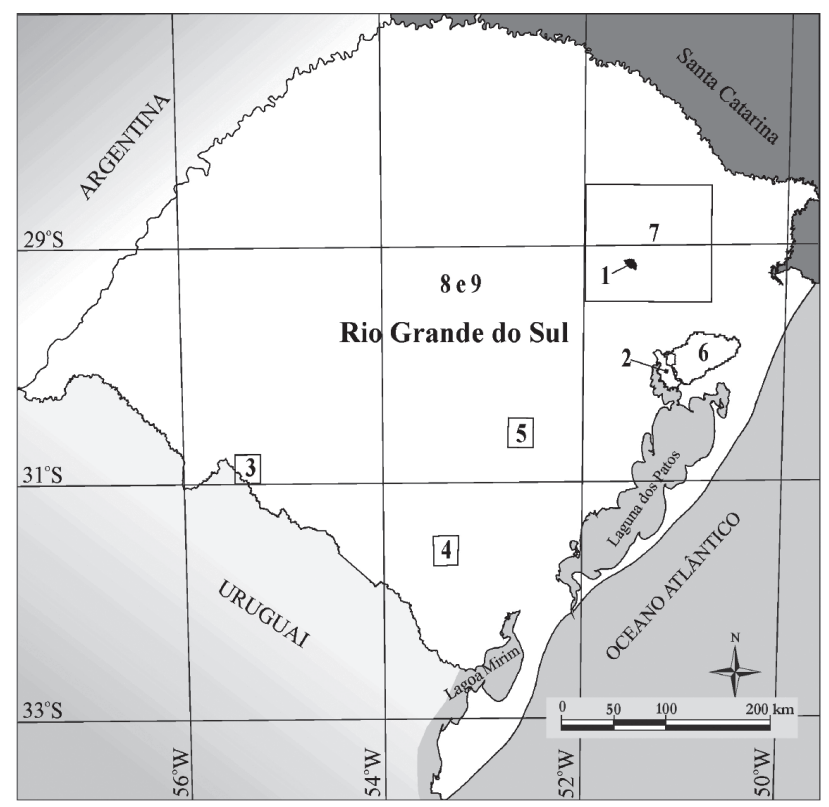

Figura 1. Localização de mapas convencionais de solos do Rio Grande do Sul, elaborados nas escalas 1:10.000 a 1:750.000 (1: Vale dos Vinhedos; 2: Município de Porto Alegre; 3: Folha Palomas; 4: Folha Pinheiro Machado; 5: Folha Encruzilhada do Sul; 6: bacia do Rio Gravataí; 7: Serra Gaúcha; e 8 e 9: Rio Grande do Sul). 
processamento e análise dos mapas digitalizados e georreferenciados foi realizado com auxílio do software ArcGIS (ESRI, 2006).

Inicialmente, calcularam-se a área e o perímetro de cada polígono e, a partir dessas medidas, determinaram-se a escala cartográfica efetiva dos mapas de solos e o índice de complexidade de forma. Segundo Hengl \& Husnjak (2006), a escala efetiva é definida como uma medida objetiva que indica se o entrelaçamento dos polígonos no mapa de solos corresponde à sua escala nominal, a escala de apresentação. A escala efetiva foi calculada pela seguinte equação (Forbes et al., 1987):

$$
\mathrm{ESN}=\mathrm{NSN} \times(\mathrm{IMR} / 2)
$$

em que $E S N$ é o divisor da escala efetiva; $N S N$, o divisor da escala nominal; e $I M R$, o índice de máxima redução, o fator pelo qual a escala do mapa pode ser reduzida até que o tamanho médio dos polígonos delimitados $(A S D)$ seja igual à área mínima mapeável $(M L D)$, que no Brasil é especificada como $0,4 \mathrm{~cm}^{2}$ (IBGE, 2007). O valor de $M L D$ foi previamente convertido de $\mathrm{cm}^{2}$ para seu tamanho equivalente em ha, considerando a escala nominal de cada mapa.

$A S D$ e $I M R$ foram calculadas pelas seguintes equações (Forbes et al., 1987):

$$
\begin{gathered}
A S D=\sum_{j=1}^{m} A j / m \\
I M R=\sqrt{A S D / M L D}
\end{gathered}
$$

em que $A j$ é a área do polígono $j$ (em ha) e $m$ o número total de polígonos no mapa.

O índice de complexidade de forma $(S)$ procura descrever a geometria geral dos polígonos nos mapas de solos e consiste em uma relação entre perímetro e área de cada polígono, que foi calculada pela seguinte equação (Hole, 1978):

$$
S=P / 2 r \pi, \text { em que } r=\sqrt{A / \pi}
$$

em que $P$ é o perímetro do polígono; $A$, a área do polígono; e $r$, o raio de um círculo com área igual à área do polígono em questão, todas expressas em unidades de grandeza equivalente; nesse caso, $\mathrm{m}^{2}$ para $A$ e m para $P$ e $r$. Valores de $S$ próximos a 1 caracterizam polígonos bastante compactos e simples, enquanto os mais distantes correspondem a formas mais complexas (polígonos mais estreitos ou ramificados), o que em geral significa acurácia posicional e escala efetiva maiores (D'Avello \& McLeese, 1998).

Por último, a partir da legenda de cada mapa, quantificaram-se o número de classes taxonômicas (do primeiro ao quarto nível) e o número de tipos de solos, convencionando-se "tipo de solo" como a descrição completa, incluindo as características ou propriedades que diferenciam os solos após o quarto nível de classificação taxonômica, tais como fases de relevo e pedregosidade, atividade de argila e tipo de horizonte superficial, entre outros. Adicionalmente, calcularamse também o número total de unidades de mapeamento, o número de unidades simples e combinadas e a fração da área total representada por dados não uniformes, pela seguinte equação (Nussbaum et al., 2011):

$$
P_{N D}=\left(A_{N D} / A_{M}\right) \times 100
$$

em que $P_{N D}$ é a fração com dados não uniformes; $A_{N D \text {, }}$ a área ocupada por dados não uniformes (unidades de mapeamento combinadas), e $A_{M}$, a área total mapeada.

\section{RESULTADOS}

A escala efetiva calculada em todos os mapas foi inferior à sua escala nominal (Quadro 2). Curiosamente, as menores diferenças entre as duas escalas ocorreram nos mapas 1 e 9, cujas escalas de

\begin{tabular}{|c|c|c|c|c|c|}
\hline \multirow[t]{2}{*}{ Mapa } & \multirow[t]{2}{*}{ Local/abrangência } & \multicolumn{2}{|c|}{ Área mapeada } & \multirow[t]{2}{*}{ Escala nominal } & \multirow[t]{2}{*}{ Fonte } \\
\hline & & ha & $\mathrm{km}^{2}$ & & \\
\hline 1 & Vale dos Vinhedos & $8.121,6$ & 81,2 & $1: 10.000$ & Flores et al. (2012) \\
\hline 2 & Município de Porto Alegre & $47.561,1$ & 475,6 & 1:50.000 & Schneider et al. (2008) \\
\hline 3 & Folha Palomas ${ }^{(1)}$ & $62.621,1$ & 626,2 & $1: 50.000$ & Flores et al. (2007a) \\
\hline 4 & Folha Pinheiro Machado ${ }^{(1)}$ & $65.779,1$ & 657,8 & $1: 50.000$ & Flores et al. (2007b) \\
\hline 5 & Folha Encruzilhada do Sul ${ }^{(1)}$ & $66.418,9$ & 664,2 & $1: 50.000$ & Flores et al. (2009) \\
\hline 6 & Bacia do rio Gravataí & $201.253,0$ & $2.012,5$ & $1: 50.000$ & Jungblut (1994) \\
\hline 7 & Serra Gaúcha & $1.348 .961,3$ & $13.489,6$ & $1: 50.000$ & Flores et al. (2007c) \\
\hline 8 & Rio Grande do Sul & $28.204 .501,6$ & $282.045,0$ & $1: 250.000$ & SAA/IBGE (2003) \\
\hline 9 & Rio Grande do Sul & $28.204 .501,6$ & $282.045,0$ & $1: 750.000$ & Machado \& Fitz (2001) \\
\hline
\end{tabular}
apresentação são, respectivamente, a maior e a menor do conjunto de mapas utilizado (Quadro 1). Ambos

Quadro 1. Identificação, local, área mapeada, escala nominal e fonte de mapas convencionais de solos do Rio Grande do Sul, elaborados nas escalas 1:10.000 a 1:750.000

(1) Nome da folha de acordo com a articulação das cartas topográficas da Diretoria de Serviço Geográfico (DSG) do Exército, na escala 1:50.000. 
também apresentaram o menor IMR, com valores igualmente próximos, o que demonstra que, embora elaborados em escalas de apresentação muito distantes, os dois mapas possuem consistência semelhante quanto à generalização, com poucos polígonos excessivamente grandes. Destaca-se também o mapa 8 , cuja escala nominal é numericamente três vezes maior que a do mapa 9; entretanto, sua escala efetiva dele resultou muito próxima da obtida para o último. O IMR alto confirma que o primeiro é consideravelmente mais generalizado do que faz supor sua escala nominal.

Entre os mapas elaborados na escala 1:50.000, o mapa 2 apresentou a escala efetiva mais próxima da nominal enquanto os mapas 6 e 7 apresentaram as maiores discrepâncias entre as duas escalas. O IMR evidencia que o tamanho dos polígonos nesses dois mapas está acima do ideal para sua escala de apresentação, o que pode ser produto tanto de simplificação das unidades de mapeamento quanto de dificuldade de individualização de solos com ocorrência muito intrincada. Os três mapas restantes apresentaram diferenças com magnitude semelhante entre a escala efetiva e a nominal, com valores de IMR igualmente próximos entre si.

Todos os mapas apresentaram polígonos com dimensão inferior à área mínima mapeável (MLD) para sua escala nominal, com o maior número no mapa 1, seguido dos mapas 8 e 9. Entre os mapas na escala nominal 1:50.000, o mapa 2 apresentou a maior frequência de casos, em parte devido à delimitação de Tipos de Terreno, que são áreas geralmente pequenas. O mapa 4 discriminou a menor quantidade de polígonos menores que a área mínima mapeável, e os demais oscilaram em torno de valores intermediários próximos.

O menor e o maior índice de complexidade de forma $(S)$ foram obtidos respectivamente para os mapas $1 \mathrm{e}$ 8 (Quadro 2). A ASD pequena e os polígonos inferiores à MLD explicam o baixo $S$ do primeiro, pois ambos tendem a formas mais simples. Por outro lado, o desviopadrão de $S$ evidencia certa homogeneidade na delimitação dos polígonos em todo o mapa, provavelmente em razão do uso de geotecnologias (Sarmento et al., 2008). No segundo, uma inspeção visual evidenciou que os polígonos possuem bordas bastante sinuosas e recortadas, o que resultou no maior $S$, apesar da ASD grande. O contrário foi observado no mapa 9, cujos polígonos têm bordas bem mais simplificadas, mesmo sendo sua escala efetiva próxima à do anterior. Entre os mapas 1:50.000, o mapa 2 apresentou o menor $S$, denotando maior simplificação que os demais, que apresentaram $S$ próximo entre si. Todavia, segundo a classificação proposta por Hole (1978), todos os mapas se enquadraram na mesma categoria: polígonos com forma moderadamente complexa.

Considerando-se o conjunto de mapas utilizados (Quadro 3), o número de classes taxonômicas e de tipos de solos foi aproximadamente proporcional à escala e extensão da área mapeada. No caso dos mapas 8 e 9 , o número de classes taxonômicas e de tipos de solos foram praticamente idênticos, o que é coerente com suas escalas efetivas próximas (Quadro 2). O mapa 6 se diferenciou dos demais por apresentar classes taxonômicas apenas até o terceiro nível, o que pode restringir certos usos por causa da impossibilidade de inferir determinadas propriedades dos solos.

O mapa 8 apresentou o maior número de unidades de mapeamento, contrastando com o mapa 9, com menos de metade daquele. A diferença de unidades combinadas e PND entre ambos é ainda mais acentuada, o que revela estratégia distinta na delimitação da ocorrência dos solos, apesar de cobrirem a mesma área, terem escala efetiva semelhante e praticamente o mesmo número de classes taxonômicas e de tipos de solos. O mapa 1 foi o segundo mais numeroso em unidades de mapeamento por causa de

Quadro 2. Indicadores computados a partir dos polígonos de mapas convencionais de solos do Rio Grande do Sul, elaborados nas escalas $1: 10.000$ a 1:750.000

\begin{tabular}{|c|c|c|c|c|c|c|c|c|c|c|c|}
\hline \multirow[t]{2}{*}{ Mapa } & \multirow{2}{*}{$\begin{array}{c}\text { Escala } \\
\text { nominal }\end{array}$} & \multirow{2}{*}{$\begin{array}{c}\mathrm{N}^{\mathrm{o}} \mathrm{de} \\
\text { polígono }\end{array}$} & \multirow[t]{2}{*}{ ASD } & \multirow[t]{2}{*}{ MLD } & \multirow[t]{2}{*}{ IMR } & \multirow{2}{*}{$\begin{array}{l}\text { Escala } \\
\text { efetiva }\end{array}$} & \multirow{2}{*}{$\begin{array}{l}\text { Políg. } \\
\text { <MLD }\end{array}$} & \multicolumn{4}{|c|}{ Índice de complexidade de forma (S) } \\
\hline & & & & & & & & Média & Mín. & Máx. & DP \\
\hline & & & 1 & & & & & & & & \\
\hline 1 & $1: 10.000$ & 1648 & 4,9 & 0,4 & 3,51 & $1: 17.550$ & 223 & 1,87 & 1,02 & 13,72 & 0,96 \\
\hline 2 & 1:50.000 & 228 & 208,8 & 10 & 4,57 & $1: 114.229$ & 41 & 2,07 & 1,06 & 11,16 & 1,25 \\
\hline 3 & $1: 50.000$ & 159 & 393,8 & 10 & 6,28 & $1: 156.892$ & 22 & 2,51 & 1,01 & 11,92 & 1,94 \\
\hline 4 & $1: 50.000$ & 138 & 476,7 & 10 & 6,90 & $1: 172.601$ & 3 & 2,54 & 1,03 & 6,13 & 1,14 \\
\hline 5 & $1: 50.000$ & 195 & 340,6 & 10 & 5,84 & $1: 145.904$ & 16 & 2,46 & 1,02 & 7,93 & 1,24 \\
\hline 6 & $1: 50.000$ & 126 & $1.597,3$ & 10 & 12,64 & $1: 315.955$ & 15 & 2,40 & 1,04 & 12,67 & 2,01 \\
\hline 7 & $1: 50.000$ & 1626 & 829,6 & 10 & 9,11 & 1:227.709 & 24 & 2,48 & 1,01 & 22,58 & 1,61 \\
\hline 8 & $1: 250.000$ & 1214 & $21.812,1$ & 250 & 9,34 & $1: 1.167 .586$ & 196 & 2,69 & 1,04 & 20,26 & 1,78 \\
\hline 9 & $1: 750.000$ & 832 & $32.126,5$ & 2.250 & 3,78 & 1:1.417.006 & 94 & 2,03 & 1,04 & 12,17 & 1,04 \\
\hline
\end{tabular}

ASD: área média dos polígonos; MLD: área mínima mapeável; IMR: índice de máxima redução; Políg.<MLD: número de polígonos menores que a área mínima mapeável; e DP: desvio-padrão. 
Quadro 3. Indicadores computados a partir da legenda de mapas convencionais de solos do Rio Grande do Sul, elaborados nas escalas 1:10.000 a 1:750.000

\begin{tabular}{|c|c|c|c|c|c|c|c|c|c|c|}
\hline \multirow{2}{*}{ Mapa } & \multirow{2}{*}{ Escala nominal } & \multicolumn{4}{|c|}{ Classe taxonômica } & \multirow{2}{*}{ Tipo de solo } & \multicolumn{3}{|c|}{ Unidade de mapeamento } & \multirow{2}{*}{ PND } \\
\hline & & N1 & N2 & N3 & N4 & & Total & Simples & Combinada & \\
\hline & & & & & & & & & & $\%$ \\
\hline 1 & $1: 10.000$ & 6 & 12 & 37 & 54 & 156 & 156 & 156 & 0 & 0,00 \\
\hline 2 & $1: 50.000$ & 6 & 10 & 13 & 15 & 15 & 12 & 2 & 10 & 98,31 \\
\hline 3 & $1: 50.000$ & 5 & 8 & 11 & 12 & 20 & 16 & 12 & 4 & 29,16 \\
\hline 4 & $1: 50.000$ & 3 & 6 & 8 & 8 & 41 & 34 & 14 & 20 & 66,70 \\
\hline 5 & $1: 50.000$ & 3 & 6 & 8 & 8 & 45 & 31 & 14 & 17 & 45,55 \\
\hline 6 & $1: 50.000$ & 7 & 9 & 12 & - & 27 & 12 & 5 & 7 & 83,06 \\
\hline 7 & 1:50.000 & 7 & 11 & 21 & 24 & 92 & 63 & 9 & 54 & 97,32 \\
\hline 8 & $1: 250.000$ & 13 & 23 & 37 & 44 & 45 & 183 & 46 & 137 & 80,14 \\
\hline 9 & $1: 750.000$ & 13 & 25 & 36 & 48 & 48 & 75 & 46 & 29 & 27,54 \\
\hline
\end{tabular}

N1: Ordem; N2: Subordem; N3: Grande Grupo: N4: Subgrupo; e PND: fração da área mapeada com dados não uniformes.

todas serem unidades simples, representando uma única classe taxonômica. Excetuando-se esse e o mapa 3 , nos demais predominaram unidades de mapeamento combinadas, e a PND foi aproximadamente proporcional à quantidade daquelas. A PND mais alta foi encontrada no mapa 7, explicada pela ocorrência intrincada dos solos em razão da complexidade do relevo da região (Sarmento et al., 2008).

\section{DISCUSSÃO}

Os mapas utilizados neste estudo constituem uma amostra reduzida dos produtos cartográficos de levantamentos convencionais de solos executados nas diversas regiões do Brasil. Eles foram utilizados pela simples razão de estarem acessíveis aos autores em formato digital compatível com o uso imediato em SIG, sem qualquer outro critério de seleção. No entanto, o conjunto é representativo da situação da massa de dados legados de solos no Brasil e em vários outros países: um mosaico de mapas em escalas variadas, cobrindo diferentes paisagens e extensões geográficas, elaborados em épocas diferentes e a partir de materiais básicos, metodologias e recursos tecnológicos distintos, bem como executado por equipes diversas.

Os resultados evidenciaram que mapas legados de solos tendem a apresentar qualidade inferior à presumida para sua escala de apresentação. Mesmo em mapas com a mesma escala nominal, a escala efetiva apresentou variação considerável. A magnitude das diferenças observadas foi similar às encontradas por Hengl \& Husnjak (2006), em cartas de solos 1:50.000 de várias paisagens na Croácia, e por Geng et al. (2010), em um conjunto maior e mais diversificado de mapas de solos de províncias do Canadá. O enquadramento de todos os mapas na categoria de forma moderadamente complexa, independente da escala nominal, reproduz o que foi verificado por Hole (1978), no Estado norte-americano de Wisconsin, e por Hengl \& Husnjak (2006), na Croácia.

A proporcionalidade observada do número de classes taxonômicas e de tipos de solos com a escala e o tamanho da área mapeada é esperada, pois o incremento na escala de mapeamento exige maior intensidade de amostragem em campo, aumentando a chance de identificação de novas classes de solos. O mesmo se verifica com a expansão da área mapeada por causa da maior diversidade de ambientes e paisagens encontradas (Beckett \& Burrough, 1971; Rossiter, 2000). Já o número de unidades de mapeamento, unidades combinadas e a PND são mais dependentes das especificidades de cada levantamento. $\mathrm{O}$ uso de unidades combinadas, por exemplo, tende a crescer com a redução da escala ou com o aumento da complexidade da paisagem, porque o delineamento de classes taxonômicas individualizadas torna-se mais difícil ou demorado (Dalmolin et al., 2004; Rossiter, 2004; Geng et al., 2010). No entanto, apesar da mesma área de cobertura e da escala efetiva e do número de classes taxonômicas similares, os mapas 8 e 9 apresentaram valores contraditórios para esses itens. A predominância de unidades simples no segundo faz presumir maior refinamento na delimitação da ocorrência dos solos, porém sua escala reduzida torna a individualização de classes taxonômicas inviável. O que ocorreu é que apenas a classe dominante foi identificada, as inevitáveis inclusões de outras classes não foram explicitadas.

Os resultados demonstram a importância e necessidade de uma avaliação objetiva para orientar a tomada de decisões sobre o uso de mapas legados de solos. Mais do que desqualificar um determinado mapa, o cálculo de indicadores possibilita avaliar sua compatibilidade com os objetivos específicos de diferentes usuários (Rossiter, 2004; Malone et al., 2012). Conforme observado por Carré et al. (2007), 
isso é particularmente relevante em MDS, pois em geral as abordagens buscam otimizar a acurácia dos modelos e minimizar os erros de predição, sem a devida atenção à qualidade dos dados legados usados como entrada. A falta de avaliação prévia ou a posteriori de tais dados também é frequente em outras áreas de aplicação.

Dos indicadores utilizados, os relativos ao tamanho e à forma dos polígonos têm a vantagem da facilidade e rapidez de determinação, salvo se os mapas existirem apenas na forma impressa e necessitarem ser previamente estruturados em SIG (Dalmolin et al., 2004). A escala efetiva propicia leitura e interpretação direta, além de constituir uma informação especialmente útil para MDS porque tanto a captura da variabilidade espacial dos solos quanto a própria capacidade de predição de classes ou propriedades são dependentes da escala (Lin et al., 2005; Kim \& Zheng, 2011). O índice de complexidade de forma também fornece informação de interesse para MDS porque polígonos de bordas mais detalhadas tendem a reduzir a inclusão de solos de unidades de mapeamento adjacentes e, consequentemente, a incerteza. Polígonos menores que a área mínima mapeável também tendem a ter menos inclusões de outros solos, apesar da incerteza proporcionalmente alta no traçado de seus limites (Rossiter, 2000).

Os indicadores temáticos obtidos da legenda são igualmente relevantes, mas necessitam de interpretação mais cuidadosa. O número de unidades de mapeamento combinadas e a PND, por exemplo, dependem da estratégia definida pelos mapeadores em razão da complexidade da paisagem, do tamanho da área e do material cartográfico básico, em balanço com os recursos financeiros, o tamanho da equipe e o tempo. Consequentemente, cada levantamento acaba tendo características próprias (Beckett \& Burrough, 1971; Bie \& Beckett, 1973; Hengl \& Husnjak, 2006; Basayigit \& Senol, 2008; Geng et al., 2010). Assim, além de avaliar se um mapa é adequado para determinado propósito, esses indicadores podem ser muito úteis para comparar mapas da mesma área ou de áreas adjacentes com vistas à sua sistematização e compatibilização (Odgers et al., 2014).

Nesse sentido, tais indicadores evidenciaram que os mapas 2 e 6, que são adjacentes, requerem harmonização prévia de escala e de legenda para sua junção, haja vista as diferenças na escala efetiva, no nível de classificação taxonômica e no uso de unidades de mapeamento combinadas. Eles revelaram também que existe potencial para incrementar o detalhe espacial no mapa 8, pois o número de unidades de mapeamento, de unidades combinadas e a PND mostram que sua riqueza temática é desproporcional à escala efetiva, em comparação com o mapa 9 que cobre a mesma área. O mesmo foi constatado anteriormente para o mapa 7 por Sarmento et al. (2013). A desagregação de unidades de mapeamento combinadas empregando modelos de MDS é uma alternativa a ser considerada para esses casos (Nauman \& Thompson, 2014; Odgers et al., 2014).

As medidas propostas neste estudo possibilitaram uma caracterização rápida e objetiva e, embora contemplem poucas características, têm potencial de aplicação prática na sistematização e documentação dos dados heterogêneos de solos disponíveis no Brasil. Isso se torna importante à medida que estruturas para catalogação e disponibilização de bases de dados geoespaciais via Internet, como a Infraestrutura Nacional de Dados Espaciais (INDE), gradativamente se consolidam (Rossiter, 2004). No entanto, apesar da vantagem de depender somente dos mapas e das respectivas legendas, e da interpretação relativamente simples, elas apenas descrevem certos aspectos de um mapa de solos. A avaliação propriamente dita depende do que o usuário pretende fazer com o mapa e, para alguns propósitos, tais medidas podem ser insuficientes.

Aquelas relativas ao tamanho e à forma dos polígonos, por exemplo, não expressam a incerteza no traçado dos limites nem eventuais deficiências no georreferenciamento, comuns em mapas anteriores à disseminação de programas de SIG, receptores GPS e imagens de satélite (Rukhovich et al., 2013). Por outro lado, os indicadores derivados da legenda não expressam o quão diferentes realmente são as classes de solos, nem se os limites entre unidades de mapeamento fazem separações úteis, o que assume relevância à medida que classes distintas podem ter algumas propriedades bastante similares; por exemplo, se as classes de uma unidade de mapeamento combinada tiverem a mesma aptidão agrícola, o fato de não estarem individualizadas é irrelevante para tal avaliação. O mesmo é válido para classes em unidades de mapeamento diferentes. Nessas situações, torna-se necessário usar medidas que possibilitem caracterizar o grau de similaridade entre classes (Minasny \& McBratney, 2007; Phillips, 2013).

Existem inúmeros outros indicadores para mensurar uma diversidade de características espaciais e temáticas, além da similaridade entre classes. No entanto, muitos deles dependem da coleta de dados em campo, o que implica em disponibilidade de pessoal e de recursos, ou de métodos avançados de análise estatística, que não são de domínio da maioria dos potenciais usuários dos mapas (Rossiter, 2000; Hengl \& Husnjak, 2006; Nussbaum et al., 2011). No caso do Brasil, convém efetuar mais estudos a fim de recomendar indicadores de determinação viável e aplicação útil aos dados legados de solos.

\section{CONCLUSÕES}

1. A qualidade de mapas convencionais de solos no Rio Grande do Sul tende a ser inferior à presumida, o que também é esperado em outras regiões do Brasil em virtude das normas e recomendações em comum. 
2. As incertezas inerentes à delimitação dos polígonos e à forma de agregação das informações temáticas em unidades de mapeamento não devem ser negligenciadas.

3. A escala efetiva é uma medida simples, de interpretação direta e com grande impacto sobre as pretensões dos usuários e os resultados de modelos, sendo recomendável sua adoção rotineira para avaliar mapas convencionais de solos.

4. Indicadores calculados a partir dos polígonos e da legenda fornecem informações úteis, mas não completas; por isso, é recomendável testar outras medidas, assim como a aplicação a mapas convencionais de solos de outras áreas.

\section{LITERATURA CITADA}

BASAYIGIT, L. \& SENOL, S. Comparison of soil maps with different scales and details belonging to the same area. Soil Water Res., 3:31-39, 2008.

BECKETT, P.H.T. \& BURROUGH, P.A. The relation between cost and utility of soil surveys: IV: Comparison of the utilities of soil maps produced by different survey procedures, and at different scales. J. Soil Sci., 22:465480, 1971.

BIE, S.W. \& BECKETT, P.H.T. Comparison of four independent soil surveys by air-photo interpretation, Paphos area (Cyprus). Photogrammetria, 29:189-202, 1973.

BUI, E.N. Soil survey as a knowledge system. Geoderma, 120:17-26, 2004.

CARRÉ, F.; McBRATNEY A.B. \& MINASNY, B. Estimation and potential improvement of the quality of legacy soil samples for digital soil mapping. Geoderma, 141:1-14, 2007.

DALMOLIN, R.S.D.; KLAMT, E.; PEDRON, F.A. \& AZEVEDO, A.C. Relação entre as características e o uso das informações de levantamentos de solos de diferentes escalas. Ci. Rural, 34:1479-1486, 2004.

D'AVELLO, T.P. \& McLEESE, R.L. Why are those lines placed where they are? An investigation of soil map recompilation methods. Soil Survey Horiz., 39:119-126, 1998.

ESRI. ArcGIS 9.2. Redlands, Environmental Systems Research Institute, 2006.

FLORES, C.A.; PÖTTER, R.O.; FASOLO, P.J.; HASENACK, H. \& WEBER, E.J. Levantamento semidetalhado de solos: Folha Palomas, Estado do Rio Grande do Sul. Porto Alegre, UFRGS, 2007a. 95p.1 mapa. CD-ROM

FLORES, C.A.; PÖTTER, R.O.; HASENACK, H. \& WEBER, E. Levantamento semidetalhado dos solos na Serra do Sudeste, RS como subsídio ao zoneamento vitivinícola: Folha Pinheiro Machado. In: CONGRESSO BRASILEIRO DE CIÊNCIA DO SOLO, 31., Gramado, 2007. Anais... Gramado, Sociedade Brasileira de Ciência do Solo, 2007b. CD-ROM
FLORES, C.A.; PÖTTER, R.O.; FASOLO, P.J.; HASENACK H. \& WEBER, E. Levantamento semidetalhado de solos: Região da Serra Gaúcha - Rio Grande do Sul. Porto Alegre, UFRGS/Embrapa Clima Temperado, 2007c.

FLORES, C.A.; PÖTTER, R.O.; HASENACK, H.; WEBER, E. \& SARMENTO, E.C. Levantamento semidetalhado dos solos na Serra do Sudeste, RS como subsídio ao zoneamento vitivinícola: Folha Encruzilhada do Sul. In: CONGRESSO BRASILEIRO DE CIÊNCIA DO SOLO, 32., Fortaleza, 2009. Anais... Fortaleza, Sociedade Brasileira de Ciência do Solo, 2009. CD-ROM

FLORES, C.A.; PÖTTER R.O.; SARMENTO, E.C.; WEBER, E.J. \& HASENACK, H. Os solos do Vale dos Vinhedos. 2.ed. Brasília, Embrapa, 2012. 176p.

FORBES, T.R.; ROSSITER, D. \& van WAMBEKE, A. Guidelines for evaluating the adequacy of soil resource inventories. Ithaca, Cornell University Department of Agronomy, Soil Management Support Services (SMSS), Soil Conservation Service, USDA, 1987. 2 print. 52p. Disponível em: <http://eprints.icrisat.ac.in/8766/1/ RP\%2D02147.pdf>. Acesso em: 3 dez. 2013.

GENG, X.; FRASER, W.; VANDENBYGAART, B.; SMITH, S.; WADELL, A.; JIAO, Y. \& PATTERSON, G. Toward digital soil mapping in Canada: Existing soil survey data and related expert knowledge. In: BOETTINGER, J.L.; HOWELL, D.W.; MOORE, A.C.; HARTEMINK, A.E. \& KIENAST-BROWN, S., eds. Digital soil mapping; Progress in soil science, 2. Amsterdam, Springer, 2010. p.325-335.

HENGL, T. \& HUSNJAK, S. Evaluating adequacy and usability of soil maps in Croatia. Soil Sci. Soc. Am. J., 70:920-929, 2006

HEUVELINK, G.B.M. \& WEBSTER, R. Modelling soil variation: Past, present, and future. Geoderma, 100:269. 301, 2001.

HOLE, F.D. An approach to landscape analysis with emphasis on soils. Geoderma, 21:1-23, 1978.

HUDSON, B.D. The soil survey as a paradigm-based science. Soil Sci. Soc. Am. J., 56:836-841, 1992.

HUNTER, G.J.; WACHOWICZ, M. \& BREGT, A.K. Understanding spatial data usability. Data Sci. J., 2:79$89,2003$.

INSTITUTO BRASILEIRO DE GEOGRAFIA E ESTATÍSTICA - IBGE. Levantamento de recursos naturais (Folha SH.22 Porto Alegre e parte das Folhas SH.21 Uruguaiana e SI.22 Lagoa Mirim), Rio de Janeiro, 1986. CD-ROM

INSTITUTO BRASILEIRO DE GEOGRAFIA E ESTATÍSTICA - IBGE. Manual técnico de pedologia. 2.ed. Rio de Janeiro, IBGE, 2007. 320p.

JUNGBLUT, M. Pedologia da bacia do rio Gravataí-RS. Porto Alegre, CPRM/Metroplan, 1994. 29p. (Série Cartas Temáticas, v.2)

KIM, D. \& ZHENG, Y. Scale-dependent predictability of DEMbased landform attributes for soil spatial variability in a coastal dune system. Geoderma, 164:181-194, 2011. 
LIN, H.; WHEELER, D.; BELL, J. \& WILDING, L. Assessment of soil spatial variability at multiple scales. Ecol. Modell., 182:271-290, 2005.

MALONE, B.P.; McBRATNEY, A.B. \& MINASNY, B. Spatial Scaling for digital soil mapping. Soil Sci. Soc. Am. J., 77:890-902, 2012.

MACHADO, M.L.L. \& FITZ, P. Rio Grande do Sul. Mapa de solos. Generalização cartográfica do levantamento de reconhecimento de solos do Rio Grande do Sul - Brasil, 1973. Porto Alegre, Emater, 2001.

McBRATNEY, A.B.; MENDONÇA-SANTOS, M.L. \& MINASNY, B. On digital soil mapping. Geoderma, 117:3-52, 2003.

MILLER, B.A. The Need to continue improving soil survey maps. Soil Horiz., 53:14-15, 2012. doi:10.2136/sh12-02-0005.

MINASNY, B. \& McBRATNEY, A.B. Incorporating taxonomic distance into spatial prediction and digital mapping of soil classes. Geoderma, 142:285-293, 2007.

NAUMAN, T.W. \& THOMPSON, J.A. Semi-automated disaggregation of conventional soil maps using knowledge driven data mining and classification trees. Geoderma, 213:385-399, 2014.

NUSSBAUM, M.; ETTLIN, L.; ÇÖLTEKIN, A.; SUTER, B. \& EGLI, M. The relevance of scale in soil maps. Bull. BGS, 32:63-70, 2011.

ODGERS, N.P.; SUN, W.; McBRATNEY, A.B.; MINASNY, B. \& CLIFFORD, D. Disaggregating and harmonising soil map units through resampled classification trees. Geoderma, 214:91-100, 2014.

OMUTO, C.; NACHTERGAELE, F. \& ROJAS, R.V. State of the art report on global and regional soil information: Where are we? Where to go? Global Soil Parthership Technical Report. Roma, FAO, 2013. 69p. Disponível em: <http://www.fao.org/docrep/017/i3161e/i3161e.pdf>. Acesso em: 15 dez. 2013.
PHILLIPS, J.D. Evaluating taxonomic adjacency as a source of soil map uncertainty. Eur. J. Soil Sci., 64:391-400, 2013.

ROSSITER, D.G. Methodology for soil resource inventories. 2.ed. Enschede, ITC, 2000. 132p. Disponível em: <http:// www.itc.nl/personal/rossiter/teach/ss m/ SSM_LectureNotes2.pdf>. Acesso em: 13 jan. 2014.

ROSSITER, D.G. Digital soil resource inventories: status and prospects. Soil Use Manage., 20:296-301, 2004.

RUKHOVICH, D.I.; KOROLEVA, P.V.; KALININA, N.V.; VIL'CHEVSKAYA, E.V.; SIMAKOVA, M.S.; DOLININA, E.A. \& RUKHOVICH, S.V. State soil map of the Russian Federation: An ArcInfo version. Eur. Soil Sci., 46:225-40, 2013.

SAA/IBGE-RS/SC. Mapa de solos do Rio Grande do Sul, escala 1:250.000. Porto Alegre, SAA-RS/IBGE-Unidade SC, 2003. (Convênio Secretaria de Agricultura e Abastecimento e Instituto Brasileiro de Geografia e Estatística/Unidade Estadual de Santa Catarina)

SANCHEZ, P.A.; AHAMED, S.; CARRÉ, F.; HARTEMINK, A.E.; HEMPEL, J.; HUISING, J.; LAGACHERIE, P.; McBRATNEY, A.B.; McKENZIE, N.J.; MENDONÇASANTOS, M.L.; MINASNY, B.; MONTANARELLA, L.; OKOTH, P.; PALM, C.A.; SACHS, J.D.; SHEPHERD, K.D.; VÅGEN, T.G.; VANLAUWE, B.; WALSH, M.G.; WINOWIECKI, L. A. \& ZHANG, G.L. Digital soil map of the world. Science, 35:681-682, 2009.

SARMENTO, E.C.; FLORES, C.A.; WEBER, E.; HASENACK, H. \& POTTER, R.O. Sistema de informação geográfica como apoio ao levantamento detalhado de solos do Vale dos Vinhedos. R. Bras. Ci. Solo, 32:795-2803, 2008.

SCHNEIDER, P.; KLAMT, E.; KÄMPF, N.; GIASSON, E. \& NACCI, D. Diagnóstico ambiental de Porto Alegre: solos. In: HASENACK, H.; WEBER, E. \& MARCUZZO, S., orgs. Diagnóstico ambiental de Porto Alegre: Geologia, solos, drenagem, vegetação e ocupação. Porto Alegre, Secretaria Municipal do Meio Ambiente, 2008. p.28-43. 